\title{
openheart Identifying patients with atrial fibrillation recurrences after two pulmonary vein isolation procedures
}

\author{
Bart A Mulder (D) , ${ }^{1}$ Meelad I H Al-Jazairi, ${ }^{1}$ Federico T Magni, ${ }^{1}$ Hessel F Groenveld, \\ Robert G Tieleman, ${ }^{1}$ Ans C P Wiesfeld, ${ }^{1}$ Yong E S Tan, ${ }^{1}$ Isabelle C Van Gelder, ${ }^{1}$ \\ Michiel Rienstra (1) , ${ }^{1,2}$ Yuri Blaauw ${ }^{1}$
}

To cite: Mulder BA, Al-Jazairi MIH, Magni FT, et al. Identifying patients with atrial fibrillation recurrences after two pulmonary vein isolation procedures. Open Heart 2021;8:e001718. doi:10.1136/ openhrt-2021-001718

Received 11 May 2021 Accepted 29 November 2021

Check for updates

(C) Author(s) (or their employer(s)) 2021. Re-use permitted under CC BY-NC. No commercial re-use. See rights and permissions. Published by BMJ.

${ }^{1}$ Department of Cardiology, University Medical Centre Groningen, Groningen, The Netherlands

${ }^{2}$ Thorax Center, Cardiology, University Medical Centre Groningen, Groningen, The Netherlands

Correspondence to Dr Bart A Mulder; b.a.mulder@ umcg.nl

\section{ABSTRACT}

Introduction Pulmonary vein isolation (PVI) is an important treatment for atrial fibrillation (AF). However, many patients need more than one procedure to maintain long-term sinus rhythm. Even after two PVIs some may suffer from AF recurrences. We aimed to identify characteristics of patients who fail after two PVI procedures.

Methods and results We included 557 consecutive patients undergoing a first PVI procedure with a secondgeneration $28 \mathrm{~mm}$ cryoballoon. Follow-up procedures were performed using radiofrequency ablation targeting reconnected PVs only. Recurrent AF was defined as any episode of AF lasting $>30$ s on ECG or 24 hour Holter monitoring performed at 3, 6 and 12 months post procedure. Mean age was $59.1 \pm 10.2$ years, $383(68.8 \%)$ were male, $448(80.4 \%)$ had paroxysmal AF and the most common underlying condition was hypertension (36.6\%). A total of 140/557 (25.1\%) patients underwent redo procedure with PVI only. Of these patients 45 (32.4\%) had recurrence of AF. These patients were comparable regarding age and sex to those in sinus rhythm after one or two procedures. Multivariate logistic regression showed that non-paroxysmal AF (OR 1.08 (95\% Cl 1.01 to 1.15$)$, estimated glomerular filtration rate $(0 \mathrm{R} 0.96,95 \% \mathrm{Cl} 0.94$ to 0.99 ), bundle branch block (OR $4.17,95 \% \mathrm{Cl} 1.38$ to 12.58), heart failure (OR $4.17,95 \% \mathrm{Cl} 1.38$ to 12.58$)$ and Left Atrium Volume Index (OR 1.04, 95\% Cl 1.01 to 1.08) were associated with $\mathrm{AF}$ recurrence after two PVIs. The area under the curve for the identified risk factors was 0.74 .

Conclusions Using a PVI-only approach, recurrence of AF after two AF ablation procedures is associated with more advanced underlying disease and persistent types of AF.

\section{INTRODUCTION}

Recent guidelines consider catheter ablation as first-line treatment especially for paroxysmal atrial fibrillation (AF). ${ }^{1}$ The hallmark of $\mathrm{AF}$ ablation is isolation of the pulmonary veins (PVIs). ${ }^{2}{ }^{3}$ Unfortunately, a significant proportion of patients experience AF recurrences following PVI $(10 \%-35 \%$ in the first year) and need a redo procedure. ${ }^{45}$

\section{Key questions}

What is already known about this subject?

- Pulmonary vein isolation (PVI) is an important treatment for patients with symptomatic atrial fibrillation (AF).

- Unfortunately, many patients have a recurrence of AF after one procedure.

- Underlying risk factors increase the likelihood for a recurrence after one PVI.

What does this study add?

- This is a large cohort of patients who had a recurrence of AF after cryoballoon PVI. At redo procedure only, the pulmonary veins were addressed and no additional ablation was performed demonstrating PVI outcome after index and redo PVI-only approach.

- At multivariate analysis, analysing indicators for failure of a PVI-only approach underlying conditions (heart failure, bundle branch block, Left Atrium Volume Index, estimated glomerular filtration rate) and persistent types of AF were identified.

How might this impact on clinical practice?

- It is critical to understand which patients have long-term benefit from a strategy including PVI. Addressing the underlying conditions before the procedure might be essential to secure long-term results.

- Future PVI studies could furthermore implement these risk factors for diverse PVI strategies in different patient populations.

AF recurrences may occur due to reconnection of the pulmonary veins. Other reasons for $\mathrm{AF}$ recurrence include non-PV triggers and extensive atrial structural remodelling. ${ }^{67}$ Contributing to these recurrences of AF may be specific risk factors such as type of $\mathrm{AF}$, size of the left atrium (LA) and presence of comorbidities. $^{8-11}$ These have been implemented into risk scores that can be used to predict failure after single PVI. ${ }^{112} 13$

There is a wide variety of ablation strategies that can be performed at the redo procedure including reisolation of the PVs, non-PV 
trigger ablation and substrate ablation. ${ }^{1}{ }^{14} 15$ Recently, more extensive ablation beyond the PVI was shown to be equally effective as PVI alone in patients with persistent $\mathrm{AF}^{3}$ For this reason, it is reasonable to use a PVI ablationonly approach for paroxysmal and persistent AF. Consequently, at redo procedures, reisolation of the PVs (resulting in a higher portion of patients who will achieve $\mathrm{PV}$ isolation) is commonly performed. The evidence for additional atrial ablation is limited and atrial tachycardias/flutter may occur as a result of re-entry in incomplete lines.

Unfortunately, also after redo PVI ablation, AF recurrences occur. Since it is likely that the PVs are isolated in these patients, the AF recurrence should be explained by mechanisms beyond the PVs. These patients may potentially benefit from ablation at additional locations.

Thus, it is important to identify patients who benefit from a PVI-only approach and/or vice versa, who may be candidates for additional atrial ablation. For this purpose, we identified patients who had a recurrence of AF despite two ablation procedures targeting only the PVs. The result of the present analysis may improve future patient selection and determine AF ablation strategy.

\section{METHODS}

\section{Patient selection}

In total, 557 of 646 patients were selected from our $\mathrm{AF}$ Cryoballoon Registry at the University Medical Center Groningen. These were consecutive patients who underwent their first PVI between 2013 and 2017. To address the aim of the study, two groups of patients were identified. Group 1 included patients with a successful AF ablation outcome after the index or redo PVI procedure. Group 2 consisted of patients who had recurrence of AF despite two PV ablation-only procedures or who had already isolated PVs (no reconnections) at the redo procedure (failed PVI approach). The following patients were excluded from the analysis: patients with failed PVI who did not undergo a second PVI or patients with failed index PVI who had additional non-PVI ablation (lines, posterior wall isolation). Patients who underwent a pace and ablate strategy or a surgical AF ablation as follow-up procedure were consequently excluded. For the final analysis, we compared patients with failed PVI approach (failure after second PVI only) to successful PVI approach (figure 1). All data were retrospectively collected from the patients' medical files. All patients consented to the ablation procedures.

\section{Index PVI with cryoballoon}

The ablation procedure was performed under conscious sedation. Left atrial access was achieved with a single transseptal puncture guided by intracardiac echo and/or fluoroscopy. The target ACT level was $>300$. The secondgeneration $28 \mathrm{~mm}$ cryoballoon (Artic Front Advance, Cryocath) was used for PVI since 2014. Until 2017, at least two cryothermal applications (lasting 240s) were delivered to isolate each vein. In 2017, we adopted the approach proposed by Aryana et al. Briefly, the number of cryoapplications was limited to one if time to isolation (TTI) was $\leq 60 \mathrm{~s}$. A 'bonus' freeze was delivered if a TTI of $<60$ s was not observed. ${ }^{16}$ During cryothermal ablation

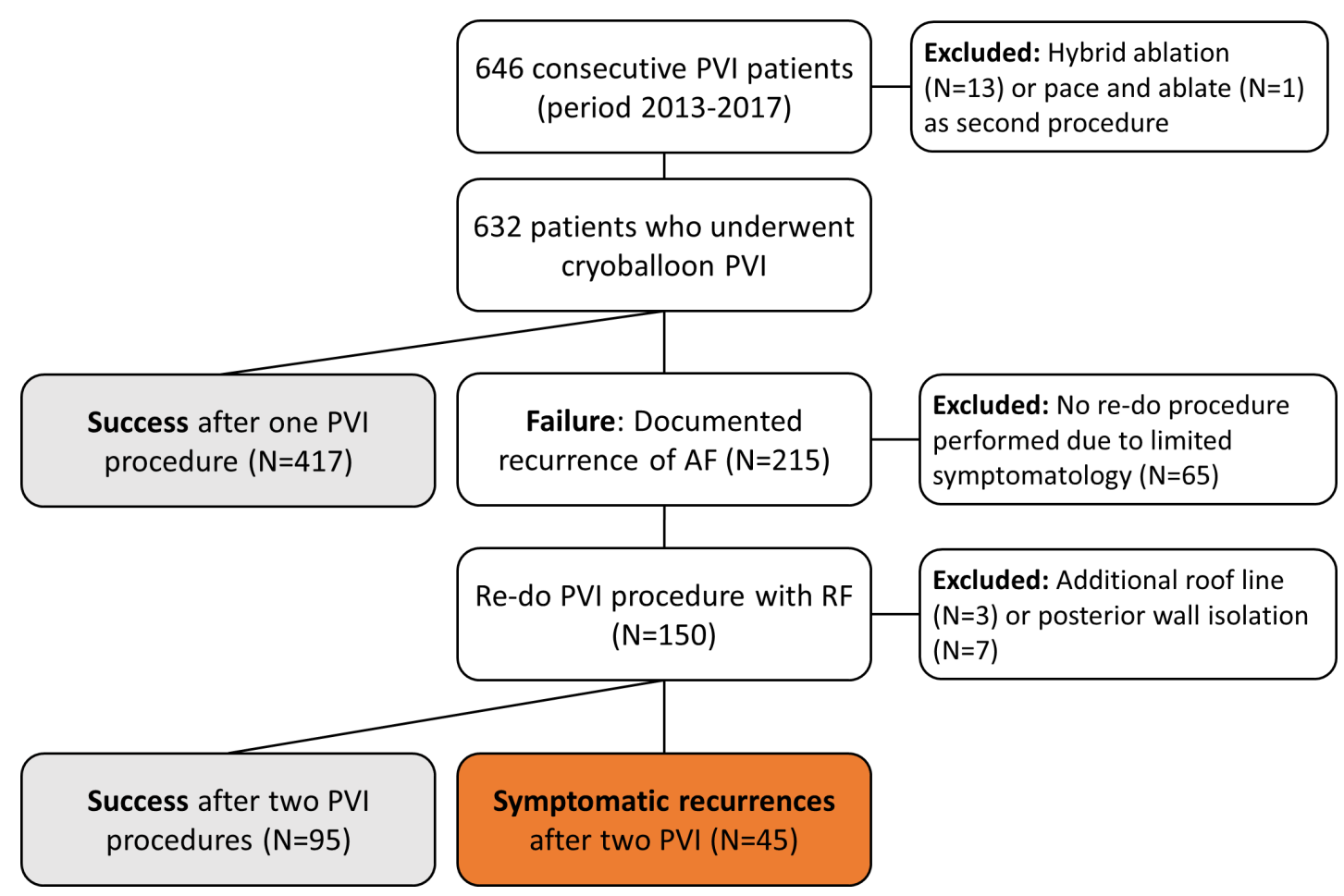

Figure 1 Flowchart of patients who underwent rhythm control strategy in the period of 2013-2017 with the start of a cryoballoon procedure. PVI, pulmonary vein isolation. 


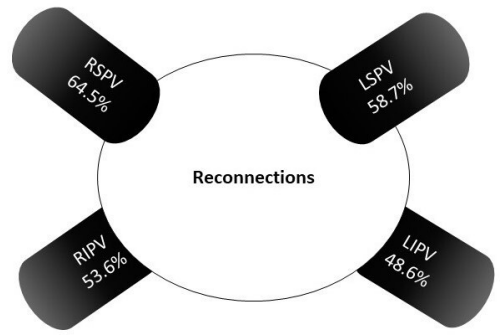

Figure 2 Percentages of reconduction found at redo procedure. LIPV, left inferior pulmonary vein; LSPV, left superior pulmonary vein; RIPV, right inferior pulmonary vein; $\mathrm{RSPV}$, right superior pulmonary vein.

of the right pulmonary veins, diaphragmatic stimulation was performed to avoid phrenic nerve injury. Electrical isolation of the pulmonary veins was evaluated using the circular Achieve mapping catheter (entrance block). Touch-up ablation with either radiofrequency ablation or cryoablation (Freezor Max) was performed in case cryoballoon ablation failed to isolate the PVs.

\section{Redo pulmonary vein ablation procedure}

For recurrences of symptomatic AF redo procedures were performed. Following double transseptal puncture mapping was performed with the EnSite Velocity or Precision (Abbott) or Rhythmia HDx (Boston Scientific) or CARTO (Biosense Webster) mapping systems. Mapping of the LA and PVs was performed with multipolar catheters: AFocus, HD Grid (Abbott), Orion catheter (Boston Scientific) or Lasso Nav or Pentaray (Biosense Webster). Ablation was performed with irrigated tip catheters: TactiCath SE (Abbott), Intellanav OI MIFI (Boston Scientific) or Smarttouch (Biosense Webster). Cardioversion was performed before left atrial mapping in case of AF. During the redo procedure, the pulmonary veins were assessed during coronary sinus pacing and gaps were consequently isolated using radiofrequency applications of $35 \mathrm{~W}$ (anterior wall) or $30 \mathrm{~W}$ (posterior wall) of $60 \mathrm{~s}$.

\section{Study outcomes}

Following all PVI procedures (index and redo PVI procedure), patients were seen in the outpatient clinic with ECG and 24 hour Holter monitoring at 3, 6 and 12 months after index procedure. In case of symptoms and absence of AF on ECG an event recorder was performed. If an unplanned hospital visit with AT/AF occurred or an episode of AT/AF lasting more than $30 \mathrm{~s}$ was documented or $\mathrm{AT} / \mathrm{AF}$ was recorded with an event recorder, during the first year of follow-up the procedure was classified as failed PVI. Recurrences occurring in the first 3 months were excluded (blanking period).

\section{Statistical analysis}

Normally distributed continuous variables were given as mean $\pm \mathrm{SD}$, skewed data as median with $\mathrm{IQR}$ and

\section{Table 1 Baseline characteristics}

\begin{tabular}{|c|c|c|c|}
\hline & Failure after two PVIs ( $\mathrm{N}=45)$ & Success after single or double PVI ( $n=512)$ & $P$ value \\
\hline Age (years), mean $\pm S D$ & $59.5 \pm 9.1$ & $58.8 \pm 10.1$ & 0.683 \\
\hline Female sex, no. (\%) & $16(35.6 \%)$ & 158 (30.9\%) & 0.506 \\
\hline Duration of AF, median (IQR) & $1540(752-2915)$ & $1318(621-2584)$ & 0.282 \\
\hline Type of AF, no. (\%) & & & 0.016 \\
\hline Paroxysmal & $32(71.1 \%)$ & 416 (81.3\%) & \\
\hline Persistent & $11(24.4 \%)$ & $93(18.2 \%)$ & \\
\hline Longstanding persistent & $2(4.4 \%)$ & $3(0.6 \%)$ & \\
\hline eGFR, mean $\pm S D$ & $75.6 \pm 15.0$ & $81.8 \pm 14.7$ & 0.009 \\
\hline Body mass index, mean $\pm S D$ & $28.1 \pm 4.9$ & $27.4 \pm 4.2$ & 0.259 \\
\hline Diabetes mellitus, no. (\%) & $1(2.2 \%)$ & $43(8.4 \%)$ & 0.141 \\
\hline Hypertension, no. (\%) & $16(35.6 \%)$ & $188(36.7 \%)$ & 0.877 \\
\hline Heart failure, no. (\%) & $7(15.6 \%)$ & $23(4.5 \%)$ & 0.007 \\
\hline Coronary artery disease, no. (\%) & $1(2.2 \%)$ & $53(10.4 \%)$ & 0.756 \\
\hline Vascular disease, no. (\%) & $2(4.4 \%)$ & $17(3.3 \%)$ & 0.660 \\
\hline Ischaemic stroke, no. (\%) & $4(8.9 \%)$ & $35(6.8 \%)$ & 0.544 \\
\hline Bundle branch block, no. (\%) & $6(13.3 \%)$ & $26(5.1 \%)$ & 0.023 \\
\hline Prior AAD, no. (\%) & $32(71.1 \%)$ & $349(68.2 \%)$ & 0.741 \\
\hline Amiodarone use, no. (\%) & $7(15.6 \%)$ & $57(11.1 \%)$ & 0.336 \\
\hline LAVI, mean \pm SD & $36.7 \pm 10.3$ & $32.9 \pm 9.2$ & 0.013 \\
\hline LVEF, mean $\pm S D$ & $53.4 \pm 5.4$ & $54.1 \pm 4.4$ & 0.352 \\
\hline
\end{tabular}

AAD, antiarrhythmic drug; AF, atrial fibrillation; eGFR, estimated glomerular filtration rate; LAVI, Left Atrium Volume Index; LVEF, left ventricular ejection fraction; PVI, pulmonary vein isolation. 
Table 2 Logistic regression for the outcome of AF recurrence after two PVIs

\begin{tabular}{|c|c|c|}
\hline & $\begin{array}{l}\text { Univariate } \\
\text { B }(95 \% \text { Cl), p value }\end{array}$ & $\begin{array}{l}\text { Multivariate* } \\
\text { B }(95 \% \mathrm{Cl}), \text { p value }\end{array}$ \\
\hline Age (years) & 1.01 (0.98 to 1.04$), p=0.683$ & \\
\hline Female sex & 1.24 (0.65 to 2.34), $p=0.515$ & \\
\hline Duration of AF & $1.00(1.00$ to 1.00$), p=0.533$ & \\
\hline Non-paroxysmal AF & 1.07 (1.00 to 1.13$), p=0.038$ & 1.08 (1.01 to 1.15$), p=0.033$ \\
\hline eGFR & 0.97 (0.95 to 0.99$), p=0.011$ & 0.96 (0.94 to 0.99$), p=0.009$ \\
\hline Body mass index & 1.04 (0.97 to 1.11$), p=0.259$ & \\
\hline Bundle branch block & 2.88 (1.12 to 7.40$), p=0.029$ & 4.17 (1.38 to 12.58$), p=0.011$ \\
\hline Diabetes mellitus & 0.24 (0.33 to 1.84$), p=0.173$ & \\
\hline Hypertension & 0.95 (0.50 to 1.80$), p=0.877$ & \\
\hline Heart failure & 3.92 (1.58 to 9.71$), p=0.003$ & 4.70 (1.49 to 14.86$), p=0.008$ \\
\hline Coronary artery disease & 0.85 (0.29 to 2.45$), p=0.757$ & \\
\hline Vascular disease & 1.35 (0.30 to 6.06$), p=0.691$ & \\
\hline CVA & 1.33 (0.45 to 3.93 ), $p=0.606$ & \\
\hline Prior AAD use & 1.15 (0.59 to 2.25$), p=0.684$ & \\
\hline Amiodarone use & 1.47 (0.63 to 3.45$), p=0.375$ & \\
\hline LAVI & 1.04 (1.01 to 1.08$), p=0.015$ & 1.04 (1.01 to 1.08$), p=0.046$ \\
\hline LVEF & 0.97 (0.92 to 1.03$), p=0.355$ & \\
\hline
\end{tabular}

${ }^{*}$ Adjusting for other baseline variables.

AAD, antiarrhythmic drug; AF, atrial fibrillation; CVA, cerebrovascular accident; eGFR, estimated glomerular filtration rate; LAVI, Left Atrium Volume Index; LVEF, left ventricular ejection fraction; PVI, pulmonary vein isolation.

categorical data as numbers with percentages. The $\chi^{2}$ was used to compare nominal variables. Univariate logistic regression was done to identify risk indicators of AF recurrence after two PVIs. All variables used in the univariate analysis were used in the multivariate model to account for confounding. Area under the curve (and the receiver operator curves) was assessed using multivariable logistic regression. All tests of significance were two sided, with p- values of $<0.05$ assumed to indicate significance. Data were analysed with Stata/SE V.16.1 (StataCorp, College Station, Texas).

\section{RESULTS \\ Patient population}

Illustrated in figure 1 is that several patients were excluded to assure patients with PVI only were included. After index PVI, 13 patients had a hybrid AF ablation as second procedure, 1 patient opted for pace and ablate and in 65 patients no redo procedure was performed (usually patient preference due to reduction of symptoms after index PVI). Furthermore, 10 patients were additionally excluded from the analysis as left atrial linear lesions in addition to PVI were made during the redo procedure. Therefore, in total 557 patients were used for the present analysis (figure 1). In these 557 patients undergoing index PVI, $31.2 \%$ were female, the average age was 59.1 years and $80.4 \%$ and $19.6 \%$ had paroxysmal and persistent AF, respectively. Following the index PVI, $417(74.9 \%)$ had sinus rhythm during 1-year follow-up and AF recurrence for which redo PVI was performed was observed in $140(25.1 \%)$. At the redo procedure, PV reconnection was observed in $2.1 \%, 60.9 \%$ and $37.0 \%$ for respectively $0,1-2$ and $\geq 3$ of the PVs. The mean number of reconnected veins was $2.2 \pm 1.0$ per patient. After the redo procedure all PVs were again isolated. Percentages of individual pulmonary veins showing reconduction at redo procedure are shown in figure 2 .

\section{AF recurrence after index or redo $\mathrm{PVI}$}

In total 557 patients were included in the analysis. In 140 (25.1\%) patients, a redo procedure was performed of which $42(30 \%)$ had a recurrence of AF after the redo procedure and in $3(2.1 \%)$ all pulmonary veins were isolated at redo PVI. Table 1 shows baseline characteristics of the patients who had AF recurrences after two PVIs versus those with sinus rhythm after index or redo PVI. There were no differences in age $(59.5 \pm 9.1$ years vs $58.8 \pm 10.1$ years, $\mathrm{p}=0.683$ ) or sex (women $16(35.6 \%)$ vs $158(30.9 \%), \mathrm{p}=0.506)$. Patients with recurrences of AF after two procedures more often had persistent $(24.4 \%$ vs $18.2 \%$ ) or long-standing persistent $\mathrm{AF}$ (4.4\% vs $0.6 \%$ ) and less often paroxysmal AF ( $71.1 \%$ vs $81.3 \%$ ) ( $\mathrm{p}$ value for group comparison=0.016) and more severe underlying disease as shown in table 1.

\section{Risk indicators of recurrence after two PVls}

Multivariate logistic regression showed that nonparoxysmal AF (OR 1.08 (95\% CI 1.01 to 1.15), estimated glomerular filtration rate (eGFR) (OR 0.96, 95\% CI 0.94 
Who fails a PVI

only approach?
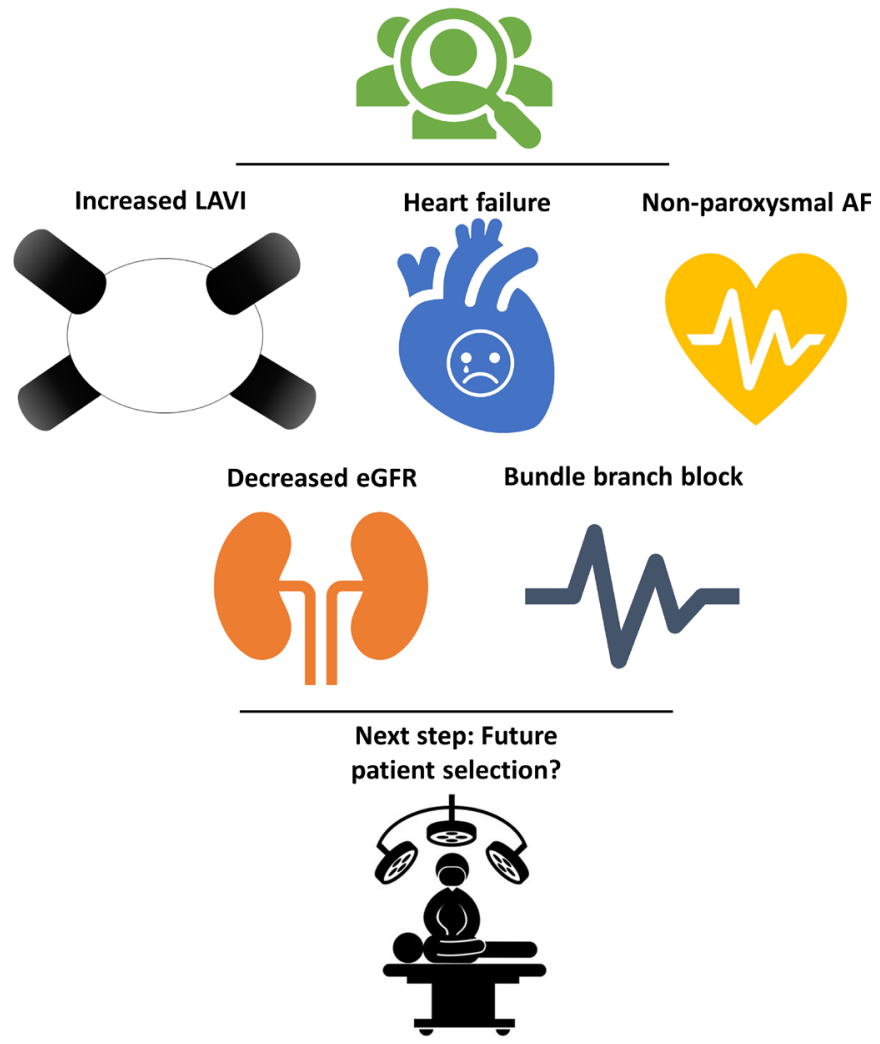

Figure 3 Central figure illustrating potential characteristics of patients in whom a pulmonary vein isolation (PVI) approach alone might be less beneficial. AF, atrial fibrillation; eGFR, estimated glomerular filtration rate; LAVI, Left Atrium Volume Index.

to 0.99 ), bundle branch block (OR 4.17, 95\% CI 1.38 to 12.58), heart failure (OR 4.17, 95\% CI 1.38 to 12.58) and Left Atrial Volume Index (LAVI) (OR 1.04, 95\% CI 1.01

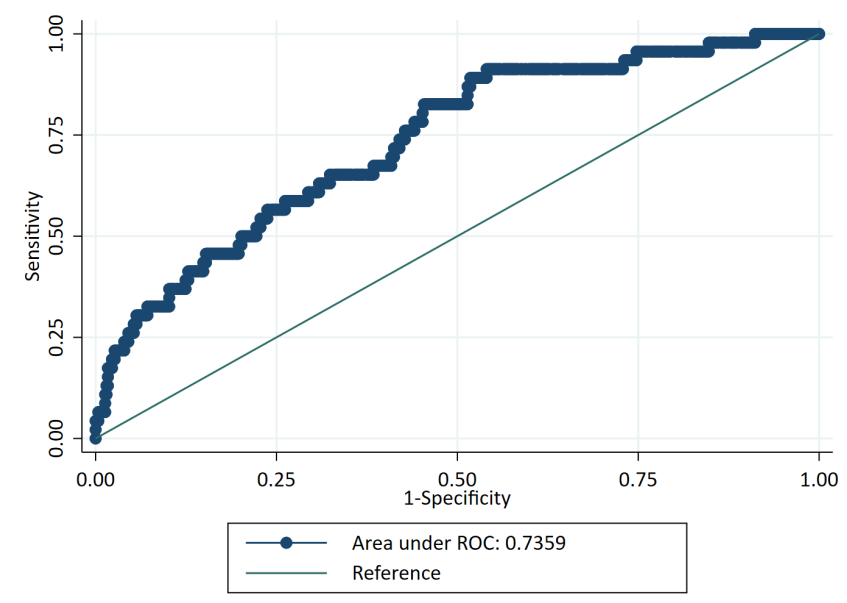

Figure 4 Receiver operator curve (ROC) for the multivariable model (including non-paroxysmal AF, estimated glomerular filtration rate, bundle branch block, heart failure, Left Atrium Volume Index which were associated with $\mathrm{AF}$ recurrence after two PVIs). AF, atrial fibrillation; PVI, pulmonary veins isolation. to 1.08) were independently associated with $\mathrm{AF}$ recurrence after two PVIs (table 2 and figure 3). The area under the curve (for all variables included in the multivariate model) as represented by receiver operator curve (ROC) is shown for the risk factors in figure 4.

\section{DISCUSSION}

In 557 patients with $\mathrm{AF}$ in whom a PVI-only ablation approach is performed (at both the index and the redo procedure), we identified several clinical risk factors associated with AF recurrences. Patients with non-paroxysmal $\mathrm{AF}$, bundle branch block, heart failure and increased LAVI had a higher chance of AF recurrence despite AF ablation procedure targeting only the PVs.

As PVI has evolved into a frontline therapy in patients with $\mathrm{AF}$, identification of patients who will not benefit from a PVI approach is pivotal. ${ }^{17} 18$ Many patients face moderate long-term outcome after single PVI. ${ }^{4}$ Although ablation technologies are improving PV reconnection remains a common phenomenon. ${ }^{19-22}$ For this reason, following $\mathrm{AF}$ recurrence, the next step is often a redo $\mathrm{AF}$ ablation procedure where the PVs are assessed and in case of reconnection conduction gaps are ablated. De Pooter et al demonstrated that many patients can be arrhythmia free following repeat PVI, highlighting the importance of obtaining durable complete PVI. ${ }^{23}$ This was also observed in the present study.

Nevertheless, as was also shown in the present study, there remains a group of patients in whom AF ablation targeting the PVs only will not be sufficient for maintaining long-term sinus rhythm. The last decades several ablation strategies targeting the atrial substrate have been introduced. These approaches include linear atrial lesions, left atrial appendage (LAA) or/and superior vena cava isolation, ablation of complex fractionated electrograms (CFAE), voltage-based approach, ablating non-pulmonary foci or ganglionated plexi or recently vein of Marshall alcohol ablation. ${ }^{141524}$ In the Substrate and Trigger Ablation for Reduction of Atrial Fibrillation Trial Part II trial, patients with persistent AF were randomised to either PVI alone (similar to our population) or PVI with addition of CFAE or PVI with addition of additional linear lines (roof and mitral valve isthmus). ${ }^{3}$ In this trial, there was no difference observed in the rate of recurrent $\mathrm{AF}$ in any of the randomised groups. ${ }^{3}$ Recently, vein of Marshall ablation in combination with LA ablation was compared with LA ablation in patients with persistent AF. Freedom from AF was slightly higher in vein of Marshall treated patients. ${ }^{24}$ Of note, these trials included patients based on the type of $\mathrm{AF}$ (persistent AF). It is likely that some of these patients had only moderate degree of structural remodelling and PV ablation only could have been sufficient. Interestingly, in the electrophysiological substrate ablation in the LA during sinus rhythm (STABLE-SR) trial, it was shown that in non-paroxysmal AF a PVI strategy including cavotricuspid isthmus ablation and targeted low-voltage areas 
and complex electrogram was equally successful as stepwise linear lesions and defragmentation. Importantly, in the substrate-based ablation group, almost half of the patients had no low voltage and only PVI ablation was performed. ${ }^{25}$ Future studies are needed to address the question whether ablation based on electroanatomical mapping or preprocedural scar characterisation with cardiac MR could potentially lead to a more tailored ablation and improve outcome.

Preprocedural predictive clinical risk factors may also be helpful to guide patient selection and ablation strategy. ${ }^{1}$ Ablation beyond the PVs should not be considered in patients with a high likelihood of maintaining sinus rhythm following ablation of only the PVs. A number of risk factors can predict $\mathrm{AF}$ recurrence after single PVI: LA size, duration of AF, age of the patient, renal dysfunction and atrial late gadolinium enhancement visualised by MRI. ${ }^{1}$ These variables are associated with structural abnormalities of the atria predisposing to more persistent form of AF. ${ }^{26}$ Kosiuk et al observed that a high APPLE Score was associated with more low-voltage areas detected during electroanatomical mapping of the LA. ${ }^{12}$ The variables found in our study suggest that patients who fail two PVIs had also already progressive disease: non-paroxysmal AF, heart failure and bundle branch block. If clinical success after two PVIs is not achievable for these patients, the question that remains is whether long-term sinus rhythm can be achieved at all or with other ablation strategies. To highlight the different ablation strategies used in populations with advanced disease, it is illustrative to observe the differences in the Catheter Ablation vs Standard Conventional Therapy in Patients with Left Ventricular Dysfunction and Atrial Fibrillation (CASTLE-AF) Study. ${ }^{27}$ In CASTLE-AF, an extended approach in addition to PVI was chosen in more than half of the patients even for the first procedure. Of the 151 patients randomised to the ablation group, in 74 patients the primary approach was PVI only and in 77 patients the first PVI was already performed with additional linear lines or CFAE. ${ }^{27}$ Importantly, at the redo procedure, 21 of the 37 patients had a redo of the PVs with additional lines. It is unknown if one approach was superior in this heart failure trial but it illustrates that many different strategies are performed as primary AF ablation approach. Another extensive ablation strategy that could be used as a primary approach in an advanced diseased population is a surgical or hybrid AF ablation. ${ }^{28}$ For example, for non-paroxysmal $\mathrm{AF}$ patients, 3-year follow-up without AF recurrences may reach up to $80 \% .{ }^{29}$ However, data in patients with heart failure are limited and ideally a randomised trial should be conducted to address the discussion which PVI approach is most successful. ${ }^{30}$ Concluding, it remains controversial in which patient's substrate ablation outside the pulmonary veins should be performed but selection may be limited to those with non-paroxysmal AF, heart failure and increased LAVI as they have an increased risk for AF recurrence after two PVIs.

\section{Limitations}

In the present analysis, we identified a subgroup of patients in whom a PVI-only approach failed. For this purpose, we identified patients who underwent at least two PVI procedures or demonstrated permanent PV isolation after the first procedure. We cannot exclude that even after the second PVI procedure, some of the veins had still no durable PVI and a third PVI procedure would have succeeded. Therefore, one cannot conclude complete PVI even after two PVI procedures. On the other hand, there is ample evidence that with each PVI the likelihood of permanent isolation increases. ${ }^{31} 32$ Recurrences of AF were monitored by standard outpatient clinic ECG and regular Holter monitoring. It is possible that patients were now classified as successful but long-term monitoring could have led to detection of asymptomatic episodes. Furthermore, more extensive follow-up would have provided greater power to our analyses and allowed us to draw more stringent conclusions on the efficacy of a PVI-only approach. Also, residual confounding may be present in the logistic regression model. To allow a robust model we did adjust for all known relevant comorbidities. There was a selection bias in our cohort as some of our (long standing) patients with persistent AF already underwent a hybrid $\mathrm{AF}$ ablation as primary approach and were not included in this paper. ${ }^{28}$ Lastly, although our analyses showed that non-paroxysmal AF, heart failure, eGFR, bundle branch block and increased LAVI are associated with a significantly higher risk of failure after a PVI-only approach, the predictive strength of these variables individually is modest.

\section{CONCLUSION}

Several clinical risk factors were identified to be associated with AF recurrence after two PVIs. These factors suggest that these patients have advanced underlying conditions. This may be used in clinical practice to identify patients in whom a PVI-only strategy may not be enough. Additional AF ablation beyond the PVI may be considered in these patients; however, further research on optimal ablation strategy in these patients is warranted.

Contributors BM, YB and MR designed the study and are responsible for the overall content as guarantor and thereby accept full responsibility for the finished work and the conduct of the study, including access to the data. BM and YB drafted the manuscript. BM performed all statistical analyses. All authors provided substantial contributions to data interpretation and critical revisions and all authors approved the final manuscript.

Funding The authors have not declared a specific grant for this research from any funding agency in the public, commercial or not-for-profit sectors.

Competing interests None declared.

Patient consent for publication Not applicable.

Ethics approval Dutch law allows the use of electronic healthcare records for research purposes under certain conditions. According to this legislation, neither obtaining informed consent from patients nor approval by a medical ethics committee is obligatory for this type of observational studies containing no directly identifiable data (Dutch Civil Law, Article 7:458).

Provenance and peer review Not commissioned; externally peer reviewed. 
Data availability statement Data are available upon reasonable request. The data that support the findings of this study are available from the corresponding author upon a reasonable request.

Open access This is an open access article distributed in accordance with the Creative Commons Attribution Non Commercial (CC BY-NC 4.0) license, which permits others to distribute, remix, adapt, build upon this work non-commercially, and license their derivative works on different terms, provided the original work is properly cited, appropriate credit is given, any changes made indicated, and the use is non-commercial. See: http://creativecommons.org/licenses/by-nc/4.0/.

\section{ORCID iDs}

Bart A Mulder http://orcid.org/0000-0002-4411-3918

Michiel Rienstra http://orcid.org/0000-0002-2581-070X

\section{REFERENCES}

1 Hindricks G, Potpara T, Dagres N. 2020 ESC guidelines for the diagnosis and management of atrial fibrillation developed in collaboration with the European association of cardio-thoracic surgery (EACTS). Eur Heart $J 2020$.

2 Haïssaguerre M, Jaïs P, Shah DC, et al. Spontaneous initiation of atrial fibrillation by ectopic beats originating in the pulmonary veins. N Engl J Med 1998;339:659-66.

3 Verma A, Jiang C-yang, Betts TR, et al. Approaches to catheter ablation for persistent atrial fibrillation. N Engl J Med 2015;372:1812-22.

4 Kuck K-H, Brugada J, Fürnkranz A, et al. Cryoballoon or radiofrequency ablation for paroxysmal atrial fibrillation. $N$ Engl $J$ Med 2016;374:2235-45.

5 Packer DL, Kowal RC, Wheelan KR, et al. Cryoballoon ablation of pulmonary veins for paroxysmal atrial fibrillation: first results of the North American Arctic front (stop AF) pivotal trial. J Am Coll Cardiol 2013;61:1713-23.

6 Shah D, Haissaguerre M, Jais P, et al. Nonpulmonary vein foci: do they exist? Pacing Clin Electrophysiol 2003;26:1631-5.

7 Qin M, Liu X, Wu S-H, et al. Atrial substrate modification in atrial fibrillation: targeting GP or CfaE? Evidence from meta-analysis of clinical trials. PLoS One 2016;11:e0164989.

8 Balk EM, Garlitski AC, Alsheikh-Ali AA, et al. Predictors of atria fibrillation recurrence after radiofrequency catheter ablation: a systematic review. J Cardiovasc Electrophysiol 2010;21:1208-16.

9 Khoueiry Z, Albenque J-P, Providencia R, et al. Outcomes after cryoablation vs. radiofrequency in patients with paroxysmal atrial fibrillation: impact of pulmonary veins anatomy. Europace 2016;18:1343-51.

10 De Maat GE, Mulder BA, Berretty WL, et al. Obesity is associated with impaired long-term success of pulmonary vein isolation: a plea for risk factor management before ablation. Open Heart 2018;5:e000771.

11 Mulder BA, Al-Jazairi MIH, Arends BKO, et al. Pulmonary vein anatomy addressed by computed tomography and relation to success of second-generation cryoballoon ablation in paroxysmal atrial fibrillation. Clin Cardiol 2019;42:438-43.

12 Kosiuk J, Dinov B, Kornej J, et al. Prospective, multicenter validation of a clinical risk score for left atrial arrhythmogenic substrate based on voltage analysis: DR-FLASH score. Heart Rhythm 2015;12:2207-12.

13 Kornej J, Hindricks G, Shoemaker MB, et al. The apple score: a novel and simple score for the prediction of rhythm outcomes after catheter ablation of atrial fibrillation. Clin Res Cardiol 2015;104:871-6.

14 Gaita F, Caponi D, Scaglione M, et al. Long-term clinical results of 2 different ablation strategies in patients with paroxysmal and persistent atrial fibrillation. Circ Arrhythm Electrophysio 2008;1:269-75

15 Mulder BA, Luermans JGLM, Hindricks G, et al. Innovations and paradigm shifts in atrial fibrillation ablation. Europace 2021;23:ii23-7.

16 Aryana A, Kenigsberg DN, Kowalski M, et al. Verification of a novel atrial fibrillation cryoablation dosing algorithm guided by time-topulmonary vein isolation: results from the Cryo-DOSING study (Cryoballoon-ablation dosing based on the assessment of Timeto-Effect and pulmonary vein isolation guidance). Heart Rhythm 2017:14:1319-25.

17 Calkins H. Catheter ablation to maintain sinus rhythm. Circulation 2012;125:1439-45.

18 Kirchhof P, Benussi S, Kotecha D. ESC guidelines for the management of atrial fibrillation developed in collaboration with EACTS: The task force for the management of atrial fibrillation of the european society of cardiology (ESC)developed with the special contribution of the european heart rhythm association (EHRA) of the ESCEndorsed by the european stroke organisation (ESO). Eur Heart $J$ 2016;2016:2893-962.

19 Kuck K-H, Fürnkranz A, Chun KRJ, et al. Cryoballoon or radiofrequency ablation for symptomatic paroxysmal atrial fibrillation: reintervention, rehospitalization, and quality-of-life outcomes in the fire and ice trial. Eur Heart J 2016;37:2858-65.

20 Phlips T, Taghji P, El Haddad M, et al. Improving procedural and oneyear outcome after contact force-guided pulmonary vein isolation: the role of interlesion distance, ablation index, and contact force variability in the 'CLOSE'-protocol. Europace 2018;20:f419-27.

21 Deisenhofer I, Estner H, Zrenner B, et al. Left atrial tachycardia after circumferential pulmonary vein ablation for atrial fibrillation: incidence, electrophysiological characteristics, and results of radiofrequency ablation. Europace 2006;8:573-82.

22 Perego GB, lacopino S, Molon G, et al. Cryoablation for paroxysmal and persistent $A F$ in patients with structural heart disease and preserved ejection fraction: clinical outcomes from 1STOP, a multicenter observational project. J Cardiol 2019;74:19-26.

23 De Pooter J, Strisciuglio T, El Haddad M, et al. Pulmonary Vein Reconnection No Longer Occurs in the Majority of Patients After a Single Pulmonary Vein Isolation Procedure. JACC Clin Electrophysiol 2019:5:295-305

24 Valderrábano M, Peterson LE, Swarup V, et al. Effect of catheter ablation with vein of Marshall ethanol infusion vs catheter ablation alone on persistent atrial fibrillation: the Venus randomized clinical trial. JAMA 2020;324:1620-8.

25 Yang B, Jiang C, Lin Y, et al. STABLE-SR (electrophysiological substrate ablation in the left atrium during sinus rhythm) for the treatment of nonparoxysmal atrial fibrillation: a prospective, multicenter randomized clinical trial. Circ Arrhythm Electrophysiol $2017 ; 10$.

26 Brandes A, Smit MD, Nguyen BO, et al. Risk factor management in atrial fibrillation. Arrhythm Electrophysiol Rev 2018;7:118-27.

27 Marrouche NF, Brachmann J, Andresen D, et al. Catheter ablation for atrial fibrillation with heart failure. N Engl J Med 2018;378:417-27.

28 Al-Jazairi MIH, Rienstra M, Klinkenberg TJ, et al. Hybrid atrial fibrillation ablation in patients with persistent atrial fibrillation or failed catheter ablation. Neth Heart J 2019;27:142-51.

29 Maesen B, Pison L, Vroomen M, et al. Three-year follow-up of hybrid ablation for atrial fibrillation. Eur J Cardiothorac Surg 2018;53:i26-32.

30 Mulder BA, Rienstra M, Van Gelder IC, et al. Update on management of atrial fibrillation in heart failure: a focus on ablation. Heart 2021. doi:10.1136/heartjnl-2020-318081. [Epub ahead of print: 04 Jun 2021].

31 Rostock T, Salukhe TV, Steven D, et al. Long-term single- and multiple-procedure outcome and predictors of success after catheter ablation for persistent atrial fibrillation. Heart Rhythm 2011;8:1391-7.

32 Ganesan AN, Shipp NJ, Brooks AG, et al. Long-term outcomes of catheter ablation of atrial fibrillation: a systematic review and metaanalysis. J Am Heart Assoc 2013;2:e004549. 